This report was prepared as an account of work sponsored by an agency of the United States Government Neither the Innted States (jovernment nor any agency thereof, nor any of their employtes, makes any warranty, express or implied, or assunes any legal liability or responsiblity for the accuracy. completeness, or usefulness of any information, apparatus, product. or process disclosed, or represents that its use would not infringe privately owned rights. Reference herein to any specific commercial product, process, or service by trade name, trademark, manufacturer, or otherwise does not necessarily constitute or imply its endorsement, recommendation, or favoring by the United States Government or any agency thereof. The views and opinions of authors expressed herein do not necessarily state or reflect those of the United States Government or any agency thereof.

\title{
PHYSICS GUIDELINES FOR THE COMPACT IGNITION TOKAMIAK
}

CONF-860652--35

\author{
M. GREENWALD. Massachusetts \\ Institute of Technology \\ Cambridge, Massachusetts 02139 \\ P. LIEWER, California Institute \\ of Technology \\ Pasadena, California 91109 \\ D. ROSS, University of Texas \\ Austin, Texas 78712 \\ C. SINGER, University of Illinois \\ Uebana. Illinois 61801 \\ H. WEITZNER, New York University. \\ New York, New York 10021
}

DE88 007276

\begin{abstract}
J. SHEFFIELD. R. A. DORY, Oax Ridge National Laboratory

P.O. Box $Y$
\end{abstract} W. A. HOULBERG. and N. A. UCKAN,

Oak Ridge. Tennessee 37831

(615) $574-1145$

M. BELL. P. COLESTOCX, J. HOSEA. S. KAYE. M. PETRAVIC. D. POST.

S. D. SCOTT, and $K$. M. YOUNG.

Princeton Plasma Physies Laboratory

Princeton, New Jersey 08544

K. H. BURRELL. N. OHYABU, and

R. STAMBAUGH. GA Technologies, Ine.

San Diego. California 92138

\begin{abstract}
The goal of the Compact Ignition Tokamais (C!T) program is to provide a cost-effective route to the production of a burning deuterium-tritium plasma, so that alpha-particle effects may be studied. A key issue to be studied in the CIT is whether alpha power behaves like other power sources in aifecting tokamak plasma confinement. The program is managed by the Princeton Plasma Physics Laboratory and includes broad community involvement. Guidelines for the preliminary design effort have been provided by the Ignition Technical Oversight Committee in discussion with the tokamak community. The reference design is a tokamak with a high fieid (10 T), high current (10 MA), a poloidal divertor. and liquid-nizrogen-cocled coils. It is a small. highpower-density device of the type proposed by Bruno Coppi (MIT). It has a major radius of $1.23 \mathrm{~m}$, a minor radius of $0.43 \mathrm{~m}$. and a plasma ellipticity of 1.8 . This paper reviews the aims of the program and the basis for the physies guidelines. The role of the CIT in the longer-term tokamak program is briefly discussed.
\end{abstract}

\section{INTRODUCTION}

The study of burning deuterium-tritium (DT) plasmas is a key element of the DOE Magnetic Fusion Program Plan.' In the present strategy, it is planned to initiate an Engineering Test Facility (ETF) in the 1990 s as an international collaborative venture. This device will perform a major long-pulse. integrated test of fusion physics and tech- nology. It is also planned to propose a smaller device, prior to the ETF, with the main goal of studying the physics of burning plasmas for short pulses and with a much smaller role in testing long-term technology. This smaller device is the Compact Ignition Tokamak (CIT), illustrated in Fig. 1. It is a concept proposed by B. Coppi ${ }^{2}$ and D. Cohn et al. ${ }^{3}$ of the Massachusetts Institute of Technology (MIT).

The sole of the CIT and its geals have been reviewed by the Magnetic Fusion Advisory Committee (MFAC) Panel XIV. The Panel made a number of findings, ${ }^{\star}$ includ. ing the following:

-Plasma behavior under ignited plasma condicions represents a new frontier of physies that must be explored and understoud as part of an assessment of magnetic fusion. Although some predicted properties of ignited plasmas can be simulated in non-ignited regimes, we also expect to uncover important new phenomena.

-During the past several years, the U.S. fusion program has examined a sequence of proposed burning-plasma tokamaks having suceessively reduced scope and cost. While giving up first. the project objecuve and nuclear-component testiug and then, very long puise operation, the program has now focussed on the CIT as a minimum-size and cost ignition exprriment that retains the capability to explore the essential tokamak burning-piasma physics issues.

-The burning-plasma issues that ate most impor. tont for the development of fusion are those relating to the confinement of the energetit-dipha par-

'Research sponsored by the Office of Fuston Energy, U.S. Department of Energy, under Centract No. DE-AC05-84OR21400 mith Martin Marietua Energy Systems. Inc. 


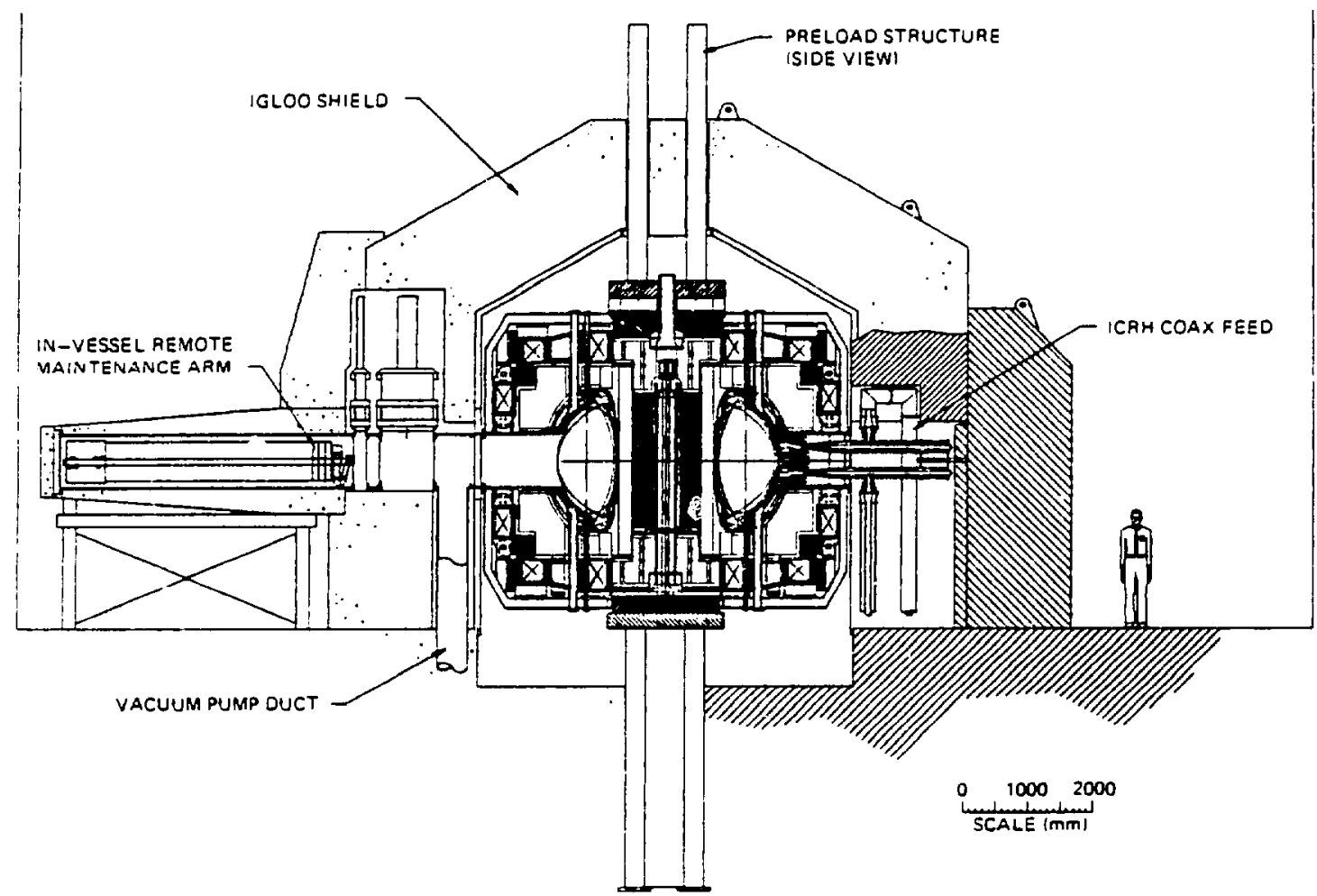

Fig. 1. Side view of the reference CTT design. Courtesy of the FEDC (ORNL).

ticles produced by the fusion reactions and the confinement of reactor-relevant plasmas that are heated mainly by these alpha particles. Other very important issues relate to controlling the profiles, thermal excursions, and composition of a burning plasma."

-The CIT Project has these primary objectives:

1. To perform a DT ignition test, including detailed studies of a burning plasma in support of the development of the tokamak reactor concept, and in support of the planning for high-duty-cycle long-pulse operation in the ETF.

2. To provide undersunding of the physics of burning plasmas which will be valuable in the development of the tokamak concept and to a lesser extent, the non-tokamak concepts.

-A secondary objective is to stumulate the development of diagnostics and remote handling for LT sysiems, and to develop generic information in areas such as pellet abiation and burn control."

Guidance for the design of the CIT has been given by the Ignition Technical Oversight Committee (ITOC). This com- mittee and the CIT physics group have been assisted in the physics area by the Ignition Physics Study Group (IPSG), which involves key tokamak experts from the U.S. laboratorio and from some foreign laboratories. The IPSG has reviewed the status of tokamaks in theory, experiment. and modeling and has assessed the guidelines for the CIT. The conclusions of the present studies are that the guidelines are sensible but that further work is desirable in a number of areas to reduce the uncertainties in extrapolations to the CIT parameters. The IPSG has made specific recommendations for work in each area. In the sections that follow the guidelines are summarized, the reference design of the CIT is given. the CITs performance is discussed, and its role in the longer-term tokamak program is described.

\section{GUIDELINES FOR THE CTT}

When calibrated in the range of currently achieved parameters, the guidelines correspond to $80 \%$ of the individual highest peformance levels. [Units used in the following discussion are: major and minor radii $R$ and $a$ in meters, current $I$ in megamperes, and magnetic tield $B$ in tesla. The cylindrical equivalent safety factor $q^{*}-2.5 a^{2} B(1+$ $\left.x^{2}\right) /[R$.] 
Energy confinement is taken to be that proposed by Goldston."

$$
1 / \tau_{E}-\left(1 / \tau_{0}^{2}+1 / \tau_{\text {zux }}^{2}\right)^{0.5} \text {. }
$$

where the ohmic component has the empirical neo-Alcator form. ${ }^{6}$

$$
T_{L}=0.07_{\pi_{30}} R^{2} a q \cdot(s) .
$$

The auxiliary heating component allows for a possible degradation of confinement with total heating power and uses the Kaye-Goldston empirical scaling. ${ }^{7}$ For limiter operation an $\mathrm{L}$-mode confinement scaling should be used,

$$
r_{\text {aus }}=0.056 I^{1.24} p^{-0.58} R^{1.65} a^{-0.49} \alpha^{0.28} \pi_{20}^{0.26} B^{-0.09} A_{i}^{0.5}(s) .
$$

For standard divertor operation an $\mathrm{H}$-mode scaling should be used. with the energy confinement time twice that of the L-mode case.

Volume-average density is constrained by the Murakami limit ${ }^{8}$ to $\left\langle n_{20}\right\rangle \leqslant 1.5 \times B / R q \cdot\left(\times 10^{20} \mathrm{~m}^{-3}\right)$ in the presence of alpha-particle heating.

Volume-average beta is held below $\langle\beta\rangle-31 / a B(\Phi)$ (the Troyon limit ${ }^{9}$ ). which corresponds to $\langle\beta\rangle=6 \%$ for typical CIT parameters.

Plasma shape is limited to moderate elongations $(\alpha \leqslant$ $1.8)$ and aspect ratios $(R / a \geqslant 2.7)$.

Plesma current is limited by requirements on the MHD safety factor, $q_{a} \geqslant 2.6$ with the auxiliary condition that $q>$ 2.0 at $0.05 \mathrm{~m}$ inside the plasma boundary.

The figure of merit $B^{2} a / q \cdot \geqslant 25 \mathrm{~T}^{2} \cdot \mathrm{m}$ in the limiter configuration.

The burn duration at maximum field and current is required to be $12 \tau_{\varepsilon}$ at $10 \mathrm{keV}$.

Impurity control is aimed at $Z_{\text {eff }} \leqslant 1.5$. A poloidal divertor is the preferred option.

Plasma heaning specifications call for a machine capability of up to $20 \mathrm{MW}$ of absorbed ion cyclotron heating (ICH) power. The projected performance with divertor operation, subject to the guidelines, requires less than this level for ignition.

The number of full-power burn pulses has been set at $\geqslant 3.000$, with the capability for $\$ 0.000$ pulses at reduced power $(7$ T. 7 MA).

\section{REFERENCE CIT}

The uriginal concept for a CIT, the IGNITOR. ${ }^{10}$ uses a pretoad on the central column of the toroidal field coils to withstand the forces. A number of alternative designs were subsequently investigated at GA Technologies (GA). MIT. Oak Ridge National Laboratory (ORNL), and Princeton
Plasma Physies Laboratory (PPPL). The reference design is a variant of the IGNITOR, developed by the Fusion Engineering Design Center (FEDC) at ORNL. which uses a hydraulic press to apply the preload. The coils are cooled by liquid nitrogen. The pulse length is constrained by coil heating. The main parameters of the CIT are listed in Table 1 .

The CIT is designed to have a poloidal divertor, and on the basis of present experiments it should be rewarded with an $\mathrm{H}$-mode form of confinement. In this circumstance the operational window is that shown by the POPCON plot" in Fig. 2a. The plot shows the contours of auxiliary power
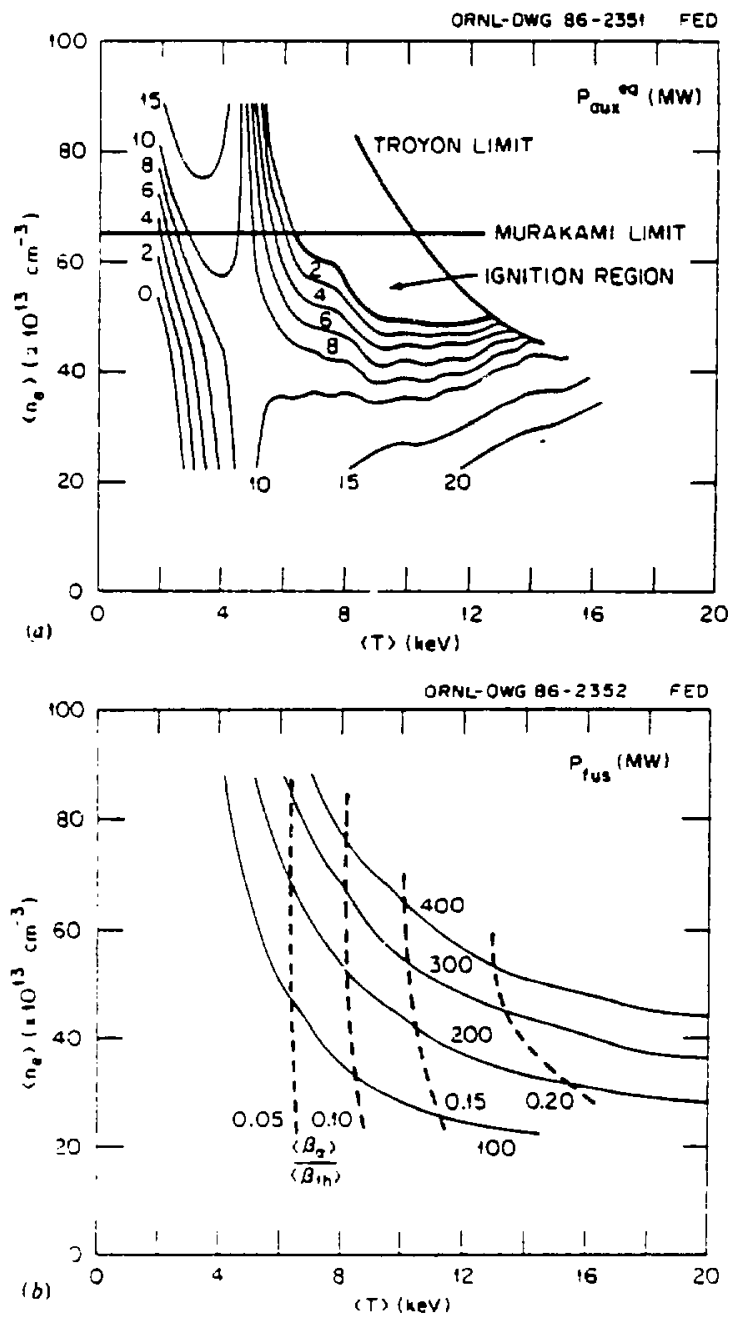

Fig. 2. (a) Curves of constant auxiliary heating power in the CIT plasme operating space with Kaye-Goldstoe $H$ mode scaling. $P_{\text {Jux }}=0$ corresponds to ignition. The operating space is constrained by the Murakami density limit and the Troyon beta limit. (b) Curves of fusion power and $<\beta_{0}>/<\beta_{1}>$. 
required to sustain the plasma at a given temperature and density. As can be seen, for this case there is a large ignition region between the ignition curve $\left(P_{\text {aur }}-0\right)$ and the beta and density limits. In $F: g$. $2 b$ a plot in $n-T$ space is given of the fusion power $\left(P_{F}\right)$ and the ratio $\left\langle\beta_{a}\right\rangle /\left\langle\beta_{t b}\right\rangle$.

Figure 3 shows a POPCON plot for the less favorable case of limiter operation with L-mode sraling. In this example the ignition window has disappeared, though if the beta and density limits are set at the best levels achieved in present-day tokamaks. ignition is recovered. The main price to pay for the less favorable scaling is the necessity to operate at a very high fusion power level, $\geqslant 300 \mathrm{MW}$.

Also shown :s the ignition curve for the limiter case in which alpha power does not cause coninement degradation. It illustrates a prime reason for building the CIT: namely, that it is critical for the optimization of DT-burning tokamaks to understand the effect of alpha power on confinement.

Eifects such as the conifinement and slowing-down of single fusion alphas may be studied in a low- $Q$. DT plasma $\left(Q-P_{F} / P_{\text {aux }}\right)$. However, the majority of important alpha effects require an ignited or high- $Q(Q>5-10)$ plasma. The burning plasma of the CIT should readily permit the study not only of single particle effects, but also of the gross effects of the alphas on stability and confinement. Of partic. ula interest are the the key issues of confinement degradation with power, thermal transients, sawtooth effects, profile evolution, ash buildup, and alpha-induced changes in impurity control. A goal of the CIT is to provide a target

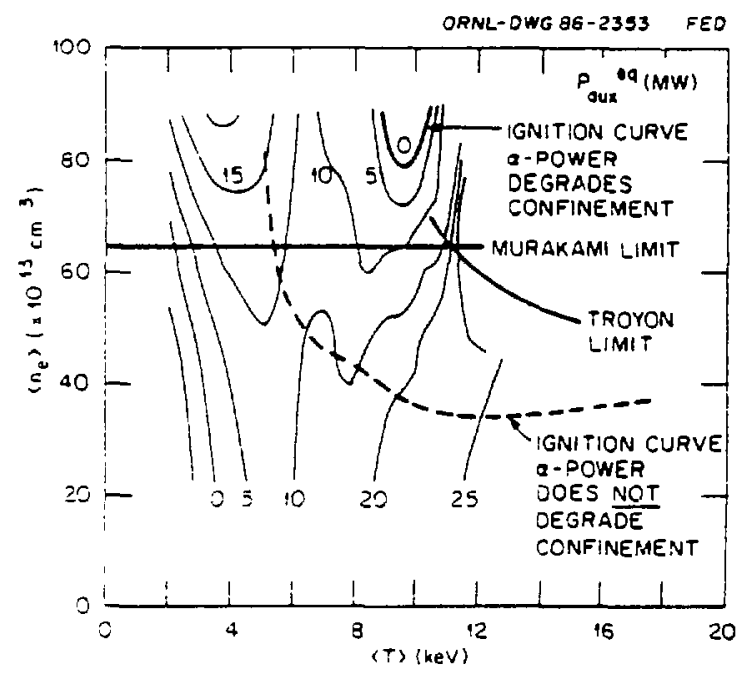

Fig. 3. Curves of constant auxiliary power in the CIT plasma operaring space for Kaye-Goldsson L-mode scaling for the case in which alphs power causes confinement degradation, and the ignition carve only for the case where alpha power does not cause confinement degradation. for the development and application of burning plasma diagnostics. The CIT, with its high neutron and gamma fluxes. is a substantial challenge for diagnostics designers.

\section{DISCUSSION OF PHYSICS GUIDELINES}

The physics guicelines for the CIT and the status of tokanak research have been reviewed by the IPSG. There is general agreement that the guidelines form a reasonable basis for the machine design. In some areas the guidelines are quite conservative; however, in a number of areas the CIT requirements represent a large extrapolation from present experience. Work is under way to reduce the uncertainties in these areas.

\section{Energy Coafinensent}

The difficulty in selecting confinement scalings is illustrated in Fig. 4, where the predictions of various confinement scalings are shown for the CIT. The uncertain performance of the CIT under the L-mode scalings, if alpha power causes confinement degradation, was one of the reasons that a poloidal divertor was chosen for base operations, though even for the most pessimistic cases the CIT should achieve $Q \geqslant 10$. For the $\mathrm{H}$-mode scalizgs the CIT should ignite with a wide margin, even in the presence of the deleterious alpha effects. A key issue is whether the $\mathrm{H}$-mode can be sustained under the CIT conditions of divertor operation, ICH and alpha power heating, and high beta and power density. In the near future, tests in tokamaks such as TFTR. DIII-D, Asdex. JET, and JT-60 should make extrapolation to the CIT more reliable.

Progress is being made theoretically in explaining the observed transport. Refinements of neoclassical ion transport theory, $^{12}$ to include finite aspect ratio and impurity effects, have led to better agreement between theory and experiment. However, experiments with ohmic heating in Alcator and with beam heating in DIII have revealed situations where the ion thermal diffusivity is well outside the range of neoclassical theory. The explanation for the anomaly may well lie in the so-called $\eta_{i}$ modes. ${ }^{13}$ which are driven by the ion temperature gradient. Figure 5 shows the improvement in ion thermal diffusivity in Alcator- $\mathrm{C}^{\text {/4 }}$ following the injection of a hydrogen pellet, which modifies the density so as to make the plasma more stable against these modes. As a rule of thumb, the modes can make the ion diffusivity, which neoclassically is small, comparable with the anomalous electron diffusivity under reactor plasma conditions. It is hoped that profile control through pellet injection can be used to mitigate this effect.

There are a number of theories to explain the anomalously high electron transport. In the ohmic region, drift-wave theories ${ }^{15}$ can match the observed behavior and explain the neo-Alcator scaling in the low- to moderatedensity regimes. Together with the $\pi_{i}$ modes, they offer a plausible explanation for the saturation in confinement at high density observed in Alcator-C and other tokamaks. 


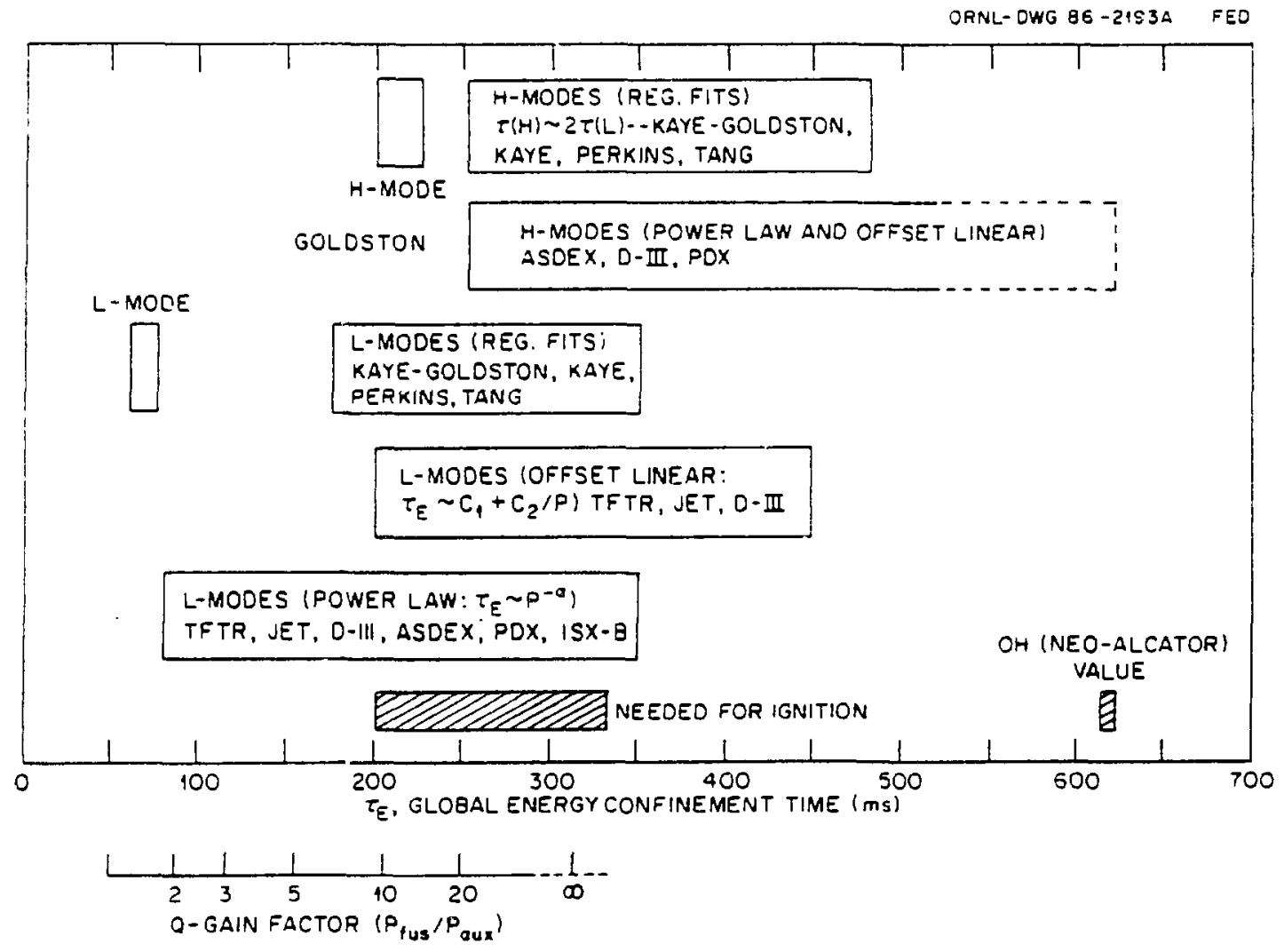

Fig. 4. Confinement projections for a reference CIT, from indiridual experiments and from regression inslysis of data from many experiments; the ignition condition depends strongly on the density profile.

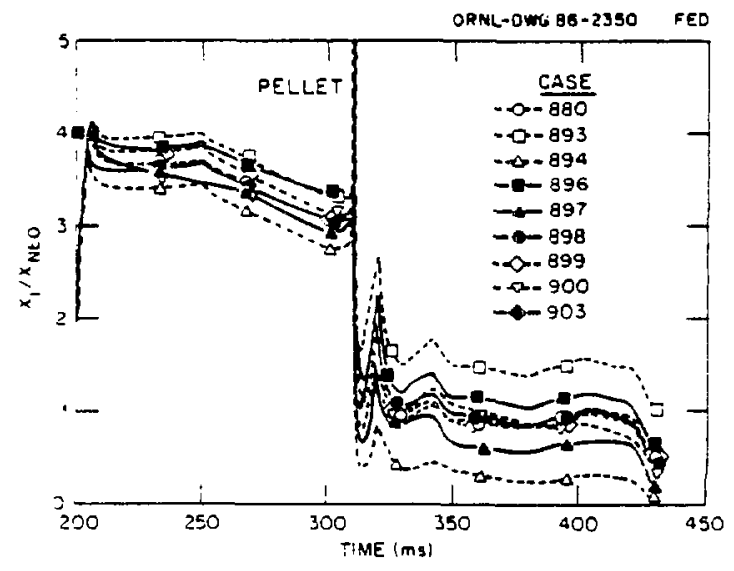

Fig. 5. Change of ion thermal diffusirity following pellet injection in Aleator- $C$, which is consistent with the suppression of ion gradient drift modes and the recovery of neo-classical ion confinement. Courtesy of S. Wolfe (MIT).
Some years ago it was observed in ISX-B that confinement degraded with increasing auxiliary power. ${ }^{16}$ In the ISX-B scaling the data could be fitted by the formula $\beta_{\mathrm{pot}} l^{0.5} \propto P^{0.33}$, and this leads to either $\tau_{E} \propto \Gamma^{1.5 p^{-0.67}}$ or $\tau_{E} \propto P^{0.5} \beta_{\text {pol }}^{-2}$. Similar results were subsequently found in other tokamaks with limiter plasmas. A regression analysis of all of these results led to the so-cailed Kaye-Goidston Lmode scaling. ${ }^{7}$ It was the Asdex group. ${ }^{17}$ studying neutralbeam-heated plasmas in divertor discharges, that discovered that if the edge electron temperature was high enough then there was much less, or no, degradation, the so-called $\mathrm{H}$ mode. Data from a number of experiments suggest that, on average, $\mathrm{H}$-mode confinement is twice as good as L-mode confinement. The plasma behavior suggests that the coniinement degradation is a result of beta effects or pronile effects caused by the heating.

Drift-wave theories, with the aid of the concept of profile consistency, ${ }^{18}$ can also reproduce some of the features of L-mode scaling in the auxiliary heating regime. In these theories the degradation would result from a transition to a 
more collisionless plasma. Experimentally, under these conditions, increases in both density and magnetic fluctuations have been observed to correlate with confinement degradation. Since the magnetic fuctuations are measured from outside the plasma, it is not known whether the internal fine-scale fluctuations are sufficient to cause the anomalous iranspor. Various theories have been given for the $\mathrm{H}$-mode effect, but none is yet generally accepted as the correct explanation. Thus, more work is required to understand the behavior of high-beta plasmas with auxiliary heating. Experiments are planned in existing tokamaks to study this area. Certainly, the uncertainties of extrapolation to the CIT plasma regime will soon be substantially reduced with the operation of a number of powerful tokamaks having massive heating capability (e.g., TFTR, DIII-D, JET, JT-60).

\section{Density Limit}

I he density limit chosen for the CIT is in the commonly ised form proposed by Murakami, ${ }^{8}\left\langle n_{20 M}\right\rangle=1.0$ $\times B / R q$. for ohmically heated plasmas and $\left\langle n_{20 M}\right\rangle=1.5$ $\times B / R q$ - for auxiliary heated plasmas. $A$ recent analysis by Greenwald (MIT) ${ }^{19}$ which takes better account of the recent data from noncircular plasmas, shows that a better empirical representation of the line density limit with auxiliary heating is $\overline{n_{20 M}}=\alpha I / \pi a b$, which may be rewritten using the definition of safety factor as $\left\langle n_{20 M}\right\rangle=1.2 B / R q_{a}[(1+$ $\left.x^{2}\right) / 2$ ]. The operating space of a number of tokamaks is shown in Fig. 6. For the CIT, with its plasma ellipticity of 1.8, the Greenwald density limit is 1.8 times higher than the guidelines, suggesting that the guideline is too conservative. However, it is not clear that much advantage can be taken

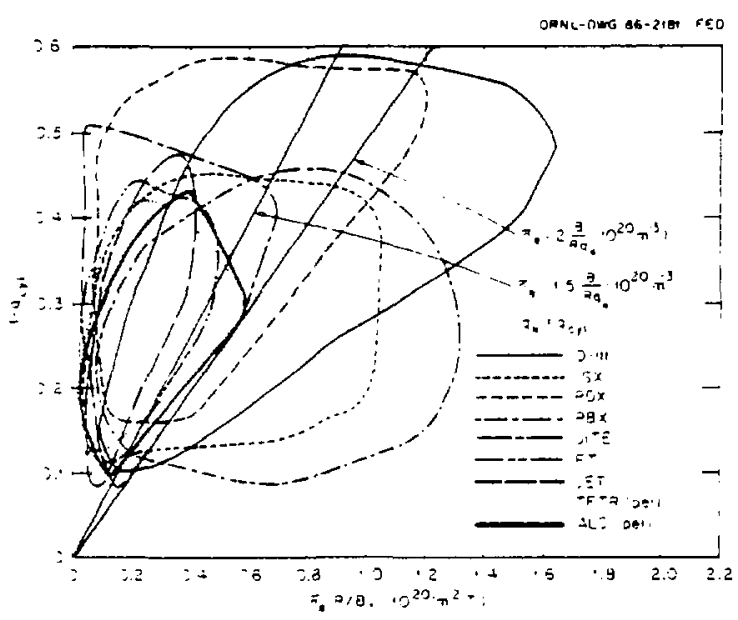

Fig. 6. The operating space in density (line density) for the Aleator-C, D-III, DITE FT, ISX-B, JET, PDX, PBX. and TFTR tokamaks. Also plotted are the ITOC guidelines for ohmic and auxiliary heated plasmas. of the higher density because of the impact of $\eta_{i}$ modes and the very large fusion powers, which must be handled at fusion temperutures.

\section{Bets Limit}

The recommended beta limit follows the Troyon scaling. ${ }^{9}\langle\beta\rangle=3.0 / / \alpha B(\%)$. It does not represent the best achieved value, as illustrated in Fig. 7. The lower level was chosen to allow a margin to avoid conditions that have been disruptive in some tokamaks. Nevertheless, distuptions are a concern for operation at the extremes of beta and density. Techniques for minimizing their occurrence will be developed in present experiments. What is not yet established is a clear-cut connection between the modes that underlie the analysis of Troyon and others and the experimentally observed modes. Work is under way to make such a connection. Other factors that are important in this area are plasma triangularity, the effect of higher ellipticity, kinetic effects at high temperature, and the question of the stability of the plasma profiles generated by alpha power.

\section{Plasma Shape and Safety Factor}

Controlled operation at plasma ellipticities up to 2 has been demonstrated in a number of tokamaks, and it will be explored further in machines such as DIII-D and JET. Vertical instabilities should not represent a problem. There is interest in operating even higher ellipticity plasmas in the CIT, and teses in DIII-D for b/a $\leqslant 2.5$ will help in the assessment of this mode.

The minimum safety factor chosen for the CIT, $q_{a} \geqslant$ 2.6, is conservative for limiter operation, though work is needed to demonstrate $\mathrm{H}$-mode operation at high beta with a divertor.

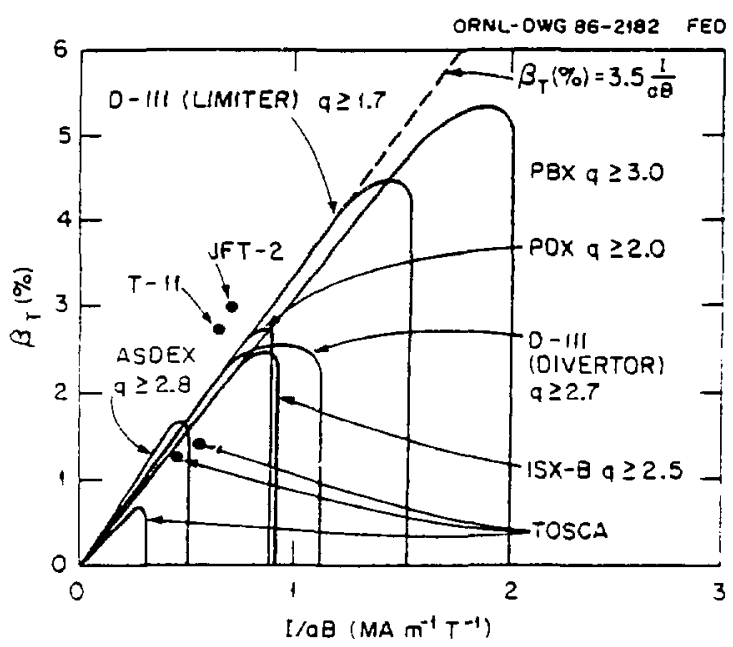

Fig. 7. Operational limits in beta of auxiliary heated tokamak exprriments. Courtesy of E. Strait (GAT). 


\section{Startup}

On the basis of data from a number of modern tokamaks it is clear that piasma initiation can be achieved with toroidal electric fields as low as $0.5-1.0 \mathrm{~V} /$ m. Therefore, plasma initiation should not present a problem for the CIT, provided adequate attention is paid to minimizing stray fields in the plasma region.

The key issue for startup is the permitted rate of rise of plasma current, since the puise length is limited by the resistive heating of the toroidal coils. With the nattop requirement of $12 r_{\varepsilon}$ and the limited duration of the toroidal field puise, it will be necessary to raise the plasma current at an average rate of $d I / d t=3 \mathrm{MA} / \mathrm{s}$. At this rate of rise, with conventional startup at constant toroidal field, there is a problem: the current does not penetrate adequately. Possible solutions are to ramp the torcidal field and current simultaneously, as illustrated in Fig. 8, or to vary the plasma shape to recuce the variation in $q_{0}$. Studies are under way on TFTR and other tokamaks to verify these techniques.

\section{Imparity Conorol}

The CIT may have to operate at very high fusion power levels, $\approx 300 \mathrm{MW}$, in order to ignite or to study alpha physics. The neutron and thermal fluxes at the plasma edge will be at r actor levels, respectively $\leqslant 8.0 \mathrm{MW} / \mathrm{m}^{2}$ and $\leqslant 2.0$ $\mathrm{MW} / \mathrm{m}^{2}$. Since the scrape-off layer thickness will be quite narrow, with $-1 / n(d n / d r)=0.01 \mathrm{~m}$ and $-1 / P(d P / d r) \approx$ $0.005 \mathrm{~m}$, and the ability to expand the scraped-off plasma

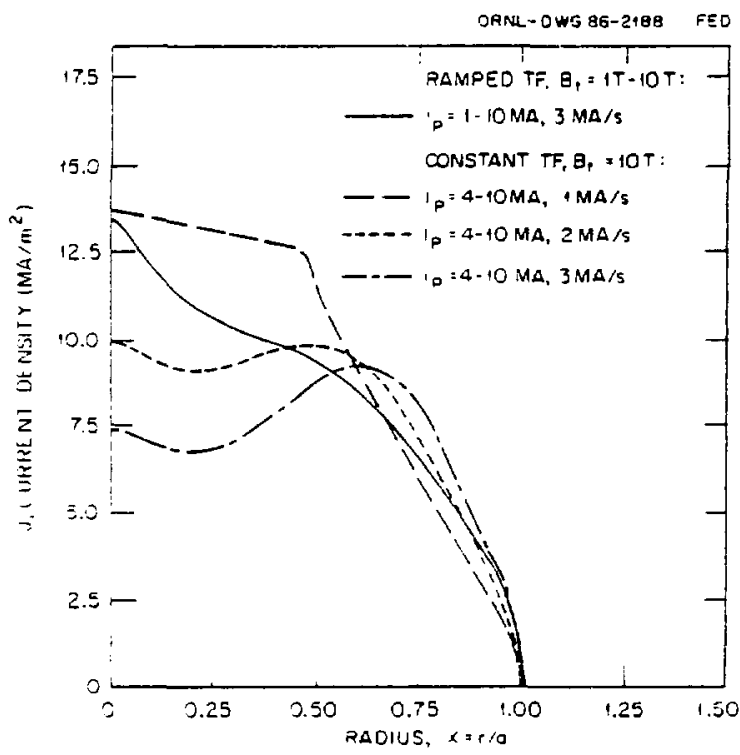

Fig. 8. Computed current density profiles for standard, constant 10-T toroidal fieid (TF), and simultaneous toroidal and poloidal fieid ramp. will be limited, the power density on the divertor targets will be high. Nevertheless, even if the thermal power density peaks at $\geqslant 10 \mathrm{MW} / \mathrm{m}^{2}$, it should be possible to handle the power with a modern divertor in which the density is high and the temperature low enough to minimize sputtering.

The additional reason for including a divertor is to allow access to the H-mode. Measurements on Asdex and D-III showed that

$$
\tau_{E} \text { (divertor) } \approx 2 \times \tau_{E} \text { (expanded boundary) } .
$$

while

$$
\left.\tau_{E}(\text { expanded boundary }) \approx 1.5 \times \tau_{\varepsilon} \text { (limiter }\right) .
$$

It is clear that programs such as those in Asdex, DIII-D, JET, JT-60, and Asdex-U, which plan to study operatior with substantial heating, including ICH and a divertor or expanded boundary, will be important to confirming confinement scalings and qualifying procedures and components for the C'T.

\section{Plasma Heating}

The choice of ICH for the CIT was made on the basis of access and cost. The compact toroidal coil system of the CIT permits only perpendicular beam injection, and because this has been obser:ed to lead to "fishbonen MHD activity, it is less satisfactory than ICH. The more compact ICH systems are preferable also for remote handling, and the total system has the lowest unit cost among the auxiliary heating options.

The CIT radial ports are sized to permit the input of up to $20 \mathrm{MW}$ of $\mathrm{ICH}$ power, though in the initial phase only $=10 \mathrm{MW}$ will be installed. Two schemes, minority and second harmonic heating, appear likely to work. While substantial plasma heating has been achieved with ICH, see Fig. 9. further work is required to optimize its application on the CIT. Of importance is a demonstration of efficient heating at the high density level of the CIT. Tests with a divertor are also important, both for demonstration of $\mathrm{H}$ mode operation at high beta and for the study of edge effects, including impurity production and removal. Experimental studies will require measurements of the ion temperature profile in order to confirm the expected deposition profile of $\mathrm{ICH}$ and to measure the ion transport coefficient. Additional effects of interest are the effect of ICH on pellets and the $\mathrm{ICH}$-induced plasma rotation.

\section{ROLE OF THE CIT IN LONG-TERM TOKAMAK PROGRAM}

Studies of ETFs, such as INTOR, NET, FER, and moce recently TIBER, ${ }^{20}$ have concentrated on poloidal divertor tokamaks with moderate aspect ratio $(\approx 2.5-4)$ strongly eiliptical $(\leqslant 1.8)$ plasmas, and superconducting coils 


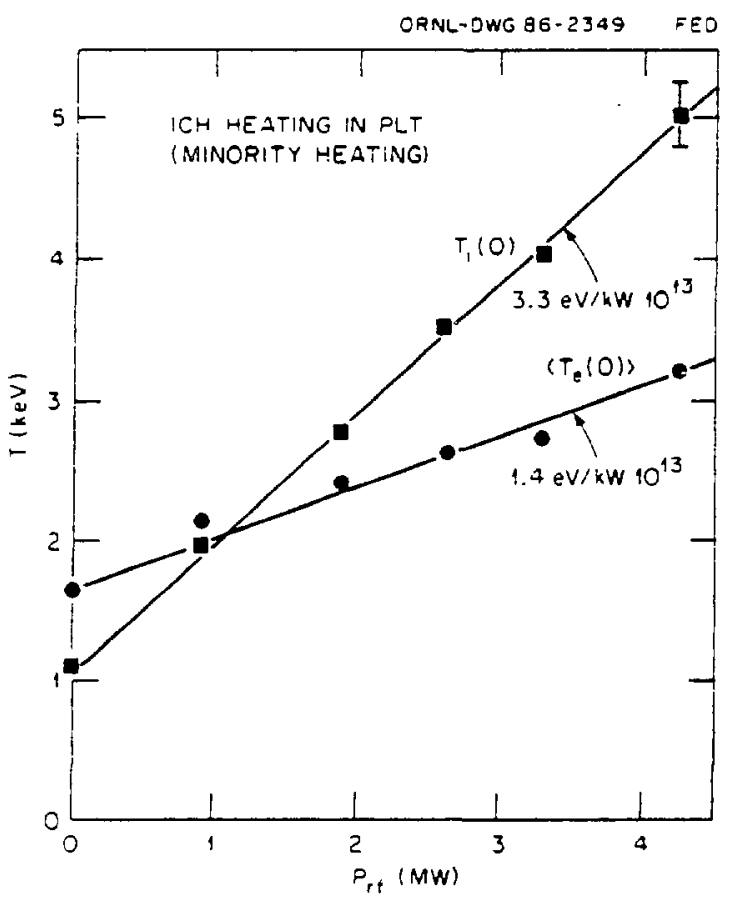

Fig. 9. ICH in PLT, which produces substantial temperature rises.

with a maximum field $\approx 10-12 \mathrm{~T}$. The present parameters of TIBER, which uses lower hybrid current drive rather than inductive drive, are listed in Table 1. The CIT, which has stmilar geometry, differs from TIBER mainly in its shorter pulse length, inductive current drive, and somewhat higher power density. An important question is, "What are the requirements for this type of configuration to make an attractive reactor, and is the CIT on the route to such a reactor?"

The question can be answered using the results of recent studies. In the Generic Reactor Studies at ORNL,"1 it was found that reactors with superconducting coils, generating some $3500 \mathrm{MW}$ of fusion power, could be economical provided the recirculating power to the plasma was $\leqslant 150$ $\mathrm{MW}(\mathrm{e})$ and the power density was high enough. The neutron flux to the first wall characterizes power density well, and $a$ value $p_{w n} \geqslant 46 \mathrm{MW} / \mathrm{m}^{2}$ is needed, the precise level depending on the other characteristics of the reactor. In another study, at Lawrence Livermore National Laboratory (LLNL), 22 it has been shown that for a lithium blanket to withstand a loss of cooling accident it is desirable to have $p_{w n} \leqslant 6-8 \mathrm{MW} / \mathrm{m}^{2}$. Consequently, a reasonable goal for an economical, inherently safe reactor is $p_{\mathrm{wn}} \approx 5-6 \mathrm{MW} / \mathrm{m}^{2}$. A second factor that affects the economics of a tokamak reactor is the shield/blanket thickness on the inboard side of the plasma. It is customary to make this as thin as possible, subject to meeting shielding and tritium blanket requirements. A commonly used value that includes the first wall and coil dewar thickness is $\Delta-1.1 \mathrm{~m}$. The fusion power for $\langle T\rangle \approx 25 \mathrm{keV}$ is given by

$$
P_{\mathrm{F}}=2.0 \times 10^{-3} a b R<\beta^{2}>B^{4}(\mathrm{MW}) \text {. }
$$

where

$$
\langle\beta\rangle \approx 3.5(1 / a B)(\%)
$$

the Troyon limit for the first stable region. The plasma current is related to the safety factor $q_{a}$ by the formula

$$
\left.I=2.75 a^{2} B\left(1+x^{2}\right) \pi q_{a} R\left(1-a^{2} / R^{2}\right)^{2}\right] .
$$

The field in the plasma $(B)$ is related to the maximum field on the toroidal coils $\left(B_{\mathrm{m}}\right)$ by

$$
B=B_{\mathrm{m}}(1.0-1.1 a / R-\mathcal{Y} R)(\mathrm{T}),
$$

where the factor 1.1 allows for a scrape-off layer.

Combining Eqs. (1) $-(3)$ with $\rho_{m a}=5.0 \mathrm{MW} / \mathrm{m}^{2}, \Delta=$ $1.1 \mathrm{~m}, B_{\mathrm{m}}=12 \mathrm{~T}$, and $P_{\mathrm{F}}=3500 \mathrm{MW}$ leads to the reactor parameters shown in Table 1.

An additional factor is the power required for current drive. Ideally, for the fast wave $P_{\mathrm{CD}}=\left(n_{20} R I / 1.4\right) \mathrm{MW}$, while for the slow wave the calculation for TIBER, ${ }^{20}$ wich is based on the analysis of Karney and Fisch. ${ }^{23}$ may be used. For the lower hybrid current drive and operation at $n_{20} \approx$ 1.1 and $\langle T\rangle \approx 25 \mathrm{keV}$, the power recirculated to support the plasma is in the range $120-190 \mathrm{MW}(\mathrm{s})$.

This analysis indicates that the CIT and TIBER are on an interesting route to an attractive reactor. The beta requirements may be met in the first stable region with the use of a plasma elongation that is close to that presently achieved and will be tested in DIII-D. The principal physics issues that are outside the CIT's capability to iest are longpulse to steady-state operation and efficient current drive. The calculations suggest that it would be interesting to test higher elongation plasmas in the CIT with $b / a \leqslant 2.4$ and $R / a=3$.

\section{CONCLUSIONS}

The physics guidelines on which the CIT is based are reasonable, given the present understanding of tokamak behavior. Work has started to improve the database, to test CIT relevant components and procedures, and, generally, to reduce the uncertainties in extrapolation to the CIT operating conditions. Key tests are to demonstrate a CIT level of current ramp, to study divertor operation and impurity control at high power density, and to optimize $[\mathrm{CH}$ under CIT-like conditions. 
Table 1. Machine and Plasma Parameters for the CIT, TIBER, and a Reactor

\begin{tabular}{llll}
\hline & \multicolumn{1}{c}{ CIT } & \multicolumn{1}{c}{ TIBER } & Example reactor \\
\hline Major radius, m & 1.22 & 2.60 & 5.2 \\
Minor radius, m & 0.45 & 0.73 & 1.6 \\
Aspect ratio & 2.7 & 3.6 & 3.25 \\
Elongation & 1.8 & 1.94 & 2.4 \\
Triangularity & 0.2 & 0.60 & \\
Toroidal field, T & 10.4 & 5.0 & 5.4 \\
Maximum field on toroidal coil, $\mathrm{T}$ & & 10 & 12 \\
Plasma current, MA & $9.0(10.0)^{a}$ & 9.7 & 20.0 \\
Edge safety factor & $2.9(2.6)$ & 2.2 & 3.0 \\
Cylindrical g- & $2.2(2.0)$ & 1.3 & 2.2 \\
Maximum density $<n>, 10^{20} \mathrm{~m}^{-3}$ & $5.8(6.5)$ & 3.3 & 8.1 \\
Critical beta. \% & $5.7(6.4)$ & 10.6 & $\mathrm{SS}$ \\
Flattop time. $\mathrm{s}$ & 3.7 & 300 & 3500 \\
Maximum fusion power, $\mathrm{MW}$ & $\approx 300$ & 440 & 5.1 \\
Maximum neutron flux, $\mathrm{MW} / \mathrm{m}^{2}$ & $6.8(8.2)$ & $1.6(4.0$ peak) & \\
Figure of merit $B^{2} a / q \circ, \mathrm{T}^{2} \cdot \mathrm{m}$ & $22.5(25)$ & & \\
\hline
\end{tabular}

Values in parentheses are for operation with a limiter rather than a divertor.

The figure of merit is proportional to $n \pi$ when $r$ is given by neo-Alcator confinement scaling and $n$ is given by the Murakami limit. It is also a convenient figure of merit for the other confinement scalings. Note that for the CIT a more conservative beta limit is used.

With $\mathrm{H}$-mode confinement and moderate impurity levels, $Z_{\text {ef }} \leqslant 1.5$, the CIT shouid ignite with a wide margin from the density or beta limits.

The CIT is on an interesting route to the realization of an attractive tokamak reactor.

\section{ACKNOWLEDGMENTS}

This paper is based upon the work of the CIT design teams. the ITOC Panel. the MFAC XIV Panch, and the TIBER Team and, in particular, on the studies undertaken by the IPSG. We appreciate the contributions of atl of these groups and, in particular, the advice of B. Coppi (MIT), $H$. Furth (PPPL, Chairman ITOC), J. Schmidt (PPPL, Project Manager CIT), R. Parker (MIT, Head of CIT Project Physics), D. Meade (PPPL, Chairman MFAC XIV), B. G. Lugan (LLNL, Leader TIBER Project). (The IPSG organization consists of three areas: Theory, headed by $H$. Weitzner (NYU): Experiment, headed by R. Stambaugh (GA); and Modeling, headed by D. Post (PPPL). The chairman and scientific secretary are, respectively, J. Sheffield and $N$. Uckan (ORNL)]. The work is coordinated in the DOE Office of Fusion Energy by C. Bolton, S. Eckstrand, T. James, P. Stone, and W. Sadowski. Valuable collaboration is under way with foreign laboratories; their IPSG representatives are $O$. Gruber (Garching, Federal Republic of Germany). P. Lallia (JET, European Economic Community), and M. Nagami (JAERI. Japan).

\section{RE.FERENCES}

1. "The Magnetic Fusion Program Plan," DOE/ER-0214, U.S. Department of Energy, Office of Fusion Energy (February 1985).

2. B. COPPI, -Compact Experiments for $\alpha$-Particle Heating," Comments Plasma Phys. Controlled Fusion, 3, 47 (1977).

3. D. COHN et al., "A High-Field Ignition Test Reactor (HFITR)," in Fusion Reactor Design Concepts. IAEA, Vienaa, pp. 113-18 (1978).

4. -Report on Assessment of Burning-Plasma Phenomena in a Compact Ignition Tokamak." presented to the Magnetic Fusion Advisory Committee by Panel XIV. February 10, 1986.

5. R. GOLDSTON, "Energy Confinement Scaling on Tokamaks," Plasma Phys. Controlled Fusion. 26. 87 (1984)

6. B. BLACKWELL et al., "Energy and Impurity Transport in the Alcator-C Tokamak." in Plasma Physics and Controlled Nuclear Fusion Research, IAEA, Vienna, Vol. II, p. 27 (1983)

7. S. M. KAYE and R. J. GOLDSTON, 'Global Energy Confinement Scaling for Neutrat-Beam Heated Tokamaks," Nucl. Fusion. 25, 65 (1985). 
8. M. MURAKAMI, J. D. CALLEN, and L. A. BERRY, -Some Observations on Maximum Densities in Tokamak Experiments," Nucl. Fusion, 16, 347 (1976).

9. F. TROYON, R. GRUBER, H. SAURENM ANN, S. SEMENZATO, and S, SUCCI, "MHD-Limits to Plasma Confinement," Plasma Phys. Controlled Fusion, 26, 209 (1984).

10. B. COPPI, "An Advanced Burning Core Experiment," PTP.84/17, Research Laboratory of Electronics. Massachusetts Institute of Technology (1984).

11. W. A. HOULBERG et al." "Contour Analysis of Fusion Reactor Plasma Performance," Nucl. Fusion, 22,935 (1982).

12. C. S. CHANG and F. L. HINTON, "Effect of Finite Aspect Ratio on the Neoclassical Ion Thermal Conductivity in the Banana Regime," Phys. Fluic's, 25, 1493 (1982).

13. B. COPPI, $M$. N. ROSENBLUTH, and R. $Z$. SAGDEEV, Phys. Fluia's. 10, 582 (1967).

14. S. WOLFE et al., "Effect of Pellet Fueling on Energy Transport in Ohmically Heated Alcator-C Plasmas." Nucl. Fusion, 26, 329 (1986).

15. F. W. PERKINS, in Heating in Toroidal Plasmas: Proceedings of the 4th International Symposium, Rome, March 1984, Vol. II, p. 977 (1984).
16. D. W. SWAIN et al, "High-Beta Injection Experiments on the ISX-B Tokamak," Nucl. Fusion, 21, 1409 (1981).

17. F. WAGNER et al, "Regime of Improved Confinement and High Beta in Neutral-Beam-Heated Discharges in the ASDEX Tokamak," Phys. Rev. Lett., 49, 1408 (1982).

18. B. COPPI, "Neoclassical Transport and the 'Principle of Profile Consistency," Comments Plasma Phys. Conrrolled Fusion, 5, 261 (1980).

19. M. GREENWALD, Massachusetts Institute of Technology, private communication (1985). To be submitted to Nuclear Fusion.

20. C. D. HENNING et al., -TIBER-Tokamak Ignition/Burn Experimental Research, Final Design Report," UCID-20589, Lawrence Livermore National Laboratory (November 1985).

21. J. SHEFFIELD et al., "Cost Assessment of a Generic Magnetic Fusion Reactor," Fusion Technol., 9, 199 (1986).

22. B. G. LOGAN, "A Rationale for Fusion Economics Based on Inherent Safety," J. Fusion Energy. 4, 245 (1985).

23. C. F. F. KARNEY and J. FISCH, -Efficiency of Current Drive by Fast Waves," PPPL-2128, Princeton Plasma Physics Laboratory (1904). 